Decentralized pricing in minimum cost spanning trees

Hougaard, Jens Leth; Moulin, Hervé; Østerdal, Lars Peter Raahave

Published in:

Economic Theory

DOI:

$10.1007 / \mathrm{s} 00199-009-0485-6$

Publication date:

2010

Document version

Publisher's PDF, also known as Version of record

Citation for published version (APA):

Hougaard, J. L., Moulin, H., \& Østerdal, L. P. R. (2010). Decentralized pricing in minimum cost spanning trees. Economic Theory, 44(2), 293-306. https://doi.org/10.1007/s00199-009-0485-6 
Econ Theory (2010) 44:293-306

DOI 10.1007/s00199-009-0485-6

RESEARCH ARTICLE

\title{
Decentralized pricing in minimum cost spanning trees
}

\author{
Jens Leth Hougaard • Hervé Moulin • \\ Lars Peter Østerdal
}

Received: 29 October 2008 / Accepted: 17 June 2009 / Published online: 4 July 2009

(C) Springer-Verlag 2009

\begin{abstract}
In the minimum cost spanning tree model we consider decentralized pricing rules, i.e., rules that cover at least the efficient cost while the price charged to each user only depends upon his own connection costs. We define a canonical pricing rule and provide two axiomatic characterizations. First, the canonical pricing rule is the smallest among those that improve upon the Stand Alone bound, and are either superadditive or piece-wise linear in connection costs. Our second, direct characterization relies on two simple properties highlighting the special role of the source cost.
\end{abstract}

Keywords Pricing rules $\cdot$ Minimum cost spanning trees $\cdot$ Canonical pricing rule . Stand-alone cost · Decentralization

\section{JEL Classification $\quad \mathrm{C} 71 \cdot \mathrm{D} 60$}

\section{Introduction}

The notion of individual guarantees is as old as the discussion of Fair Division. My actual share of the pie will depend upon other agents' characteristics, but I am

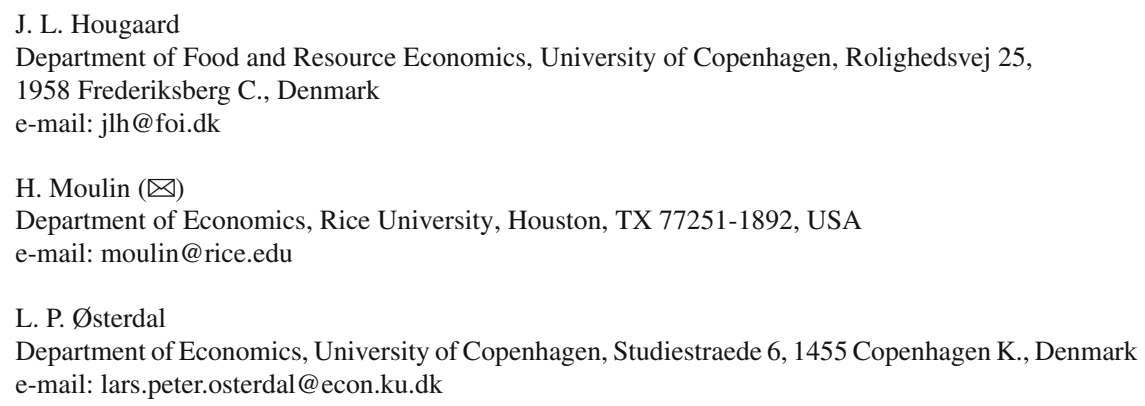


guaranteed a certain fraction of the pie no matter what these characteristics turn out to be. The higher this "worst case" share, the less risky my participation in the division rule. For instance Steinhaus (1948), and many authors after him (see e.g., Brams and Taylor 1996), regards a division of the pie as "fair" if each participant receives a share worth (to him) at least $1 / n$th of the entire pie. In the pie division problem, the $1 / n$th guarantee can be implemented, and no higher share can.

This idea has been applied to virtually all formal models of Fair Division, including public decisions with side payments (Dubins 1977; Thomson 1979; Moulin 1986), the assignment of indivisible goods (Demko and Hill 1988; Bogomolnaia and Moulin 2001) and cooperative production (Moulin 1990); see Moulin (1992) for a systematic discussion.

Here we look for the best individual guarantees in a classic network connection model, the minimal cost spanning tree (thereafter mcst) problem where a group of agents has to be connected directly or indirectly to a common supplier (source) in the least costly way (Bird 1976; Claus and Kleitman 1973; Sharkey 1995). The cost of the efficient spanning tree must be shared among the agents, and hence individual guarantees take the form of an upper bound on cost shares, and feasibility requires that the sum of these upper bounds cover at least the actual cost.

In many cost sharing problems, a natural and much discussed upper bound is the Stand Alone upper bound (e.g., Sharkey 1982; Moulin 1992), i.e., the cost of serving a given agent in the absence of other users. Its key feature is decentralization. The Stand Alone upper bound only depends upon the cost of serving the agent in question, thus it can be interpreted as a (non linear) pricing rule, that an agent can use to choose a level of demand. However, charging his Stand alone cost to every agent may be grossly inefficient. For instance in the mcst problem agent $i$ 's Stand Alone cost is that of connecting $i$ directly to the source. These charges are clearly feasible, but they ignore all potential savings from indirect connections. The challenge is to find a feasible decentralized pricing rule that improves upon (decreases significantly) the Stand Alone charges.

In some problems, the Stand Alone pricing rule cannot be improved by any other feasible decentralized pricing rule. ${ }^{1}$ We show here that in the mcst problem, a certain canonical pricing rule considerably improves upon the Stand Alone upper bound. Decentralization means here that the charge to any user only depends upon the connection costs of this particular agent to the source and to other agents; it can be computed prior to any evaluation of connection costs between other agents and between other agents and the source. The canonical charge is a simple linear combination of these costs; it is closely related to an important exact cost-sharing method for the mcst problem, introduced independently by several authors (Feltkamp et al. 1994; Norde et al. 2001; Branzei et al. 2004; Bergantinos and Vidal-Puga 1997), and dubbed the Folk solution in Bogomolnaia and Moulin (2008). The canonical charge is always

\footnotetext{
1 An example is the cooperative production problem with a one-input one-output concave cost function $C$ such that $C(0)=0$. Write $x_{i}$ for agent $i$ 's demand, and let $f\left(x_{i}\right)$ be a pricing rule improving upon the Stand Alone upper bound: $f\left(x_{i}\right) \leq C\left(x_{i}\right)$ for all $x_{i}$. Combined with feasibility, $C\left(\sum_{i} x_{i}\right) \leq \sum_{i} f\left(x_{i}\right)$, this implies easily $f \equiv C$.
} 
bounded below by that of the Folk solution, with equality whenever the cost matrix is irreducible. $^{2}$

We illustrate our pricing rule in two natural examples, one with a linear cost structure, and the other with random IID connecting costs. In both cases we find that the ratio of the total charge collected by the canonical rule to the efficient (minimal) cost grows as $\log n$ in the number $n$ of users. This compares favorably to the Stand Alone price, which in the same examples collects about $n$ times the efficient cost. Moreover the total canonical charge is a vanishing fraction of the cost of a uniform spanning tree, namely the expected cost of a spanning tree chosen uniformly among all $(n+1)^{n-1}$ spanning trees, independently of any cost consideration.

We further show that the canonical pricing rule has three desirable properties, ${ }^{3}$ pertaining to changes in connection costs and in the set of network users. The price agent $i$ pays is a continuous and weakly increasing function of agent $i$ 's connecting costs. If new users enter the network, this price decreases weakly.

Our "canonical" terminology is vindicated by three axiomatic characterizations of this pricing rule. In Theorem 1 we borrow two functional properties of the mapping from the matrix of connection costs (for all users) to the efficient cost (that of an optimal spanning tree): this mapping is superadditive and piece-wise linear. Superadditivity w.r.t. connection costs conveys the designer's preference for flexibility: it is (weakly) cheaper to build an optimal network for today's cost matrix, and possibly another network for tomorrow's cost matrix, rather than a single network optimal for the sum of today and tomorrow's connecting costs. Piece-wise linearity says that when the same network is optimal for two different cost matrices, then the optimal cost is linear in the cost matrix.

Theorem 1 states that the canonical pricing rule is the smallest one that improves upon the Stand Alone bound and is superadditive (or piece-wise linear) in the profile of connecting costs.

Theorem 2 offers an alternative characterization relying on two simple properties highlighting the special role of the source cost vis-a-vis the inter-agent connecting nodes.

\section{The MCST model}

We recall the well known minimal cost spanning tree model (see e.g. Sharkey 1995). Let $N \subset \mathbf{N}=\{1,2, \ldots$,$\} be a finite set of agents where |N|=n$. We consider networks with a source denoted by agent 0 . The source can be considered as a firm supplying the agents in $N$. A network $g$ over $N^{0}=N \cup\{0\}$ is a set of unordered pairs $i j$ where $i, j \in N^{0}$. We denote by $N^{0}(2)$ the set of such unordered pairs; its cardinality is $\frac{n(n+1)}{2}$. Sometimes we speak of $N^{0}(2)$ as the complete network on $N^{0}$. We write $G^{0}=\left\{g \mid g \subset N^{0}(2)\right\}$ for the set of all networks of $N^{0}$.

Two agents $i$ and $j$ are connected in $g$ if there is a path $i_{1} i_{2}, i_{2} i_{3}, \ldots, i_{h-1} i_{h}$ such that $i_{k} i_{k+1} \in g$ for $1 \leq k \leq h-1$ where $i=i_{1}$ and $j=i_{h}$. A network $g$ is said to

2 This means that any reduction of any connection cost decreases the optimal cost strictly. See Sect. 3.

3 They are also satisfied by the Folk solution and the Stand Alone pricing rule. 
be connected if $i$ and $j$ are connected in $g$ for all $i, j \in N^{0}$. A path is called a cycle if it starts and ends with the same agent. A network is called a tree if it contains no cycles. A spanning tree is a tree connecting all agents in $N^{0}$. There are $(n+1)^{n-1}$ such spanning trees.

For each pair $i j \in N^{0}(2)$, there is a non-negative cost $k_{i j}$ attached to the link between agents $i$ and $j$. We think of such costs as the costs of establishing the link, maintenance costs or indirect costs such as congestion, etc. The set of such costs is an element $K \in \mathbb{R}^{N^{0}(2)}$. We abuse notation by speaking of the cost matrix $K$.

A minimum cost spanning tree (mcst) is a spanning tree $T$ where the total link cost $\sum_{i j \in T} k_{i j}$ is minimized over all spanning trees of $N^{0}$. We write this minimal cost as $v(N, K)$. Note that this is also the smallest cost over all networks, not necessarily trees, connecting the source to all agents. There is a unique most if all costs $k_{i j}$ are different, but in general there may be more than one [up to $(n+1)^{n-1}$ if all costs $k_{i j}$ are equal].

Two well known algorithms for finding a minimum cost spanning tree given $K$, are due to Kruskal (1956) and Prim (1957). We recall the latter, that will be useful below. There are $n$ steps: in step 1 we pick a cheapest link between the source and one agent; in step $t$ we add one of the cheapest links between the set $M_{t-1}$ of agents already connected to the source, and $N \backslash M_{t-1}$.

\section{The canonical pricing rule: definition}

As explained in the Sect. 1, we wish to cover at least the efficient cost while charging each user of the network in a way that only depends upon his or her "local" costs.

Given a mcst problem $(N, K)$ and an agent $i$, we write $k[i]$ for the $(n-1)$ dimensional vector $\left(k_{i j}, j \in N \backslash\{i\}\right)$ of this agent's own connection costs to other agents.

Definition 1 Fix the set $N^{0}$ of agents and the source. A decentralized pricing rule is a mapping $f: \mathbb{R}_{+} \times \mathbb{R}_{+}^{n-1} \rightarrow \mathbb{R}_{+}$such that for all $x \geq 0$, the mapping $y \rightarrow f(x ; y)$ is symmetric in the $n-1$ coordinates of $y$, and for all cost matrices $K$ we have

$$
\sum_{i \in N} f\left(k_{0 i} ; k[i]\right) \geq v(N, K) .
$$

The simplest example of a decentralized pricing rule is the stand alone cost $s a\left(k_{0 i}\right.$; $k[i])=k_{0 i}$ for which inequality (1) is obvious. The canonical pricing rule defined below is unanimously preferred to $s a$ : it charges less than $s a$ to every user, often significantly so. Yet this rule too overcharges in most problems, as do all decentralized pricing rules.

Call a pricing rule budget-balanced if it always satisfies (1) with equality.

Lemma 1 For $n \geq 2$, no decentralized pricing rule is budget-balanced.

Proof Consider a problem $(N, K)$ where $k_{j l}=0$ for all $j, l \in N^{0}$. Then $f\left(k_{0 i}, k[i]\right)=0$ is the common charge of every agent. Sequentially changing the source cost from 
0 to 1 for all agents except for agent 1 and using budget-balance repeatedly, we see that the price charged to each agent cannot change. Next consider $\bar{K}$ where $\bar{k}_{0 h}=1$ for all $h \in N$ and $\bar{k}_{j l}=0$ otherwise. We have $f\left(\bar{k}_{0 i}, \bar{k}[i]\right)=0$ for all $i \neq 1$ and by budget-balance $f\left(\bar{k}_{01}, \bar{k}[1]\right)=1$. As the choice of agent 1 was arbitrary in the symmetric problem $(N, \bar{K})$, we have a contradiction.

Note that Lemma 1 also follows from Lemma 4.1(a) in Bergantinos and Vidal-Puga (2007), because our decentralized pricing rules meet the property Independence of Other Costs in that paper.

We now define the decentralized pricing rule that is the object of this paper in two equivalent ways. In Eq. 2, we denote by $\Pi_{N}$ the set of orderings of $N$. Given $\pi \in \Pi_{N}$, $\mathcal{P}(i, \pi)$ denotes the union of the source and the set of agents prior to agent $i$ in the order $\pi$, i.e. $\mathcal{P}(i, \pi)=\{0\} \cup\{j \in N \mid \pi(j)<\pi(i)\}$.

In Eq. 3 we arrange the $n-1$ numbers $k[i]$ increasingly as $k_{i}^{t}, 1 \leq t \leq n-1$, so that $k_{i}^{1} \leq \cdots \leq k_{i}^{n-1}$.

Definition 2 Given $N^{0}$, the Canonical Pricing Rule is defined as

$$
\operatorname{can}\left(k_{0 i} ; k[i]\right)=\frac{1}{n !} \sum_{\pi \in \Pi_{N}} \min _{j \in \mathcal{P}(i, \pi)}\left\{k_{i j}\right\}
$$

It is equivalently computed as

$$
\operatorname{can}\left(k_{0 i} ; k[i]\right)=\frac{1}{n} k_{0 i}+\sum_{t=1}^{n-1} \frac{1}{t(t+1)} \min \left\{k_{i}^{t}, k_{0 i}\right\}
$$

We have

$$
\operatorname{can}\left(k_{0 i} ; k[i]\right) \leq s a\left(k_{0 i} ; k[i]\right)
$$

For a given problem $(N, K)$, we compute $i$ 's canonical charge via Eq. 3, a simple linear combination after we have ordered the $n$ numbers $k_{0 i}, k_{i j}$ increasingly. Equation 2 involves an exponential number of terms, so it is not practical for computations. On the other hand it gives an intuitive interpretation of the canonical price, as the expected marginal cost of adding a given agent to a (random, and typically inefficient) spanning tree.

Pick an unbiased random ordering $\pi$ of the agents, and construct a spanning tree $T_{\pi}$ as follows. Start by connecting the first agent to the source and charging him the corresponding cost; connect next the second agent to either the source or the first agent, whichever is cheaper, and charge him that new cost;..; charge to the $t$ th agent the cost of the cheapest link to one of its predecessors or the source; and so on. This is similar to the Prim algorithm, with the crucial difference that in the latter, the $t$ th agent is not selected at random: instead it is the cheapest to connect with the $t-1$ first agents and the source. In particular for any $\pi$ the cost of $T_{\pi}$ is no less than $v(N, K)$, so inequality (1) holds. 
To see why Eqs. 2 and 3 are equivalent, fix $i=1$ for simplicity and observe that if $\left(k_{01} ; k[1]\right)$ remains in the cone in $\mathbf{R}_{+}^{n}$ such that the relative ordering of the $n$ link costs $k_{01}, k_{1 i}, 2 \leq i \leq n$ does not change, the right hand side of either equation is a linear function of $\left(k_{01} ; k[1]\right) .{ }^{4} \mathrm{~A}$ basis of such a cone is made of vectors $\left(k_{01} ; k[1]\right)$ with all coordinates equal to 0 or 1 , thus it is enough to check the equivalence for such vectors. If $k_{01}=0$, both equations give $\operatorname{can}\left(k_{01} ; k[1]\right)=0$; if $k_{01}=1$ but $k[1]=0$, both equations give $\operatorname{can}\left(k_{01} ; k[1]\right)=\frac{1}{n}$. Assume next $k_{01}=1, k_{1 i}=0$ for $2 \leq i \leq t, k_{1 j}=1$ for $t+1 \leq j \leq n$. Then $\min _{j \in \mathcal{P}(1, \pi)}\left\{k_{1 j}\right\}=1$ if and only if in the ordering $\pi$ agent 1 precedes the $t-1$ agents for which $k_{1 i}=0$. This happens with probability $\frac{1}{t}$, so (2) yields $\operatorname{can}\left(k_{01} ; k[1]\right)=\frac{1}{n !} n ! \frac{1}{t}=\frac{1}{t}$. Now (3) gives the same conclusion since

$$
\operatorname{can}\left(k_{01} ; k[1]\right)=\frac{1}{n}+\sum_{h=t}^{n-1} \frac{1}{h(h+1)}=\frac{1}{n}+\sum_{h=t}^{n-1}\left(\frac{1}{h}-\frac{1}{h+1}\right)=\frac{1}{t}
$$

We turn to the relationship between the canonical pricing rule and the (budgetbalanced) Folk solution of the mcst problem mentioned in the introduction. The latter uses a reduction of the cost matrix introduced by Bird (1976) and Aarts and Driessen (1993). Given $(N, K)$, the reduced cost matrix $K^{*}$ is the minimal matrix $K^{\prime} \leq K$ such that $v(N, K)=v\left(N, K^{\prime}\right)$. Equivalently $k_{i j}^{*}$ is the largest number $z$ such that any path from $i$ to $j$ contains at least one edge with cost at least $z$. The matrix $K$ is called irreducible if $K=K^{*}$. A matrix $K$ is irreducible if and only if for all $i, j, l$ we have $\max \left\{k_{i j}, k_{j l}\right\} \geq k_{i l}$, which is easy to recognize numerically. Moreover for any $K$, irreducible or not, the reduced matrix $K^{*}$ is irreducible.

Proposition 1 in Bogomolnaia and Moulin (2008) shows that for any irreducible cost matrix $K$, the canonical charges (can $\left.\left(k_{0 i} ; k[i]\right), i \in N\right)$ result in budget-balance. Hence the following equation

$$
\varphi_{i}(N, K)=\operatorname{can}\left(k_{0 i}^{*} ; k^{*}[i]\right) \text { for all } N, K \text { and } i
$$

defines a budget-balanced solution $\varphi$, and this is the Folk solution. Because $K^{*} \leq$ $K$, and the mapping $K \rightarrow \operatorname{can}\left(k_{0 i} ; k[i]\right)$ is monotonic, the Folk solution is always bounded above by the canonical upper bound:

$$
\varphi_{i}(N, K) \leq \operatorname{can}\left(k_{0 i} ; k[i]\right) \text { for all } i, \text { all } K
$$

with equality if the matrix $K$ is irreducible. Note however that the equality $\varphi_{i}(N, K)=$ $\operatorname{can}\left(k_{0 i} ; k[i]\right)$ for all $i$ does not imply that $K$ is irreducible. ${ }^{5}$

\footnotetext{
4 This is the piece-wise linearity property formally defined in the second paragraph of Sect. 6

5 An example is $N=\{1,2\}, k_{12}=2, k_{0 i}=1, i=1,2$. Here $v(N, K)=2=\operatorname{can}_{1}+\operatorname{can}_{2}$, yet $k_{12}^{*}=1$. 


\section{The canonical pricing rule: examples and efficiency properties}

In our two examples we compare the total charge $\sum_{N} \operatorname{can}\left(k_{0 i} ; k[i]\right)$ to three other costs: the optimal cost $v(N, K)$; the the cost of a uniform spanning tree $U(N, K)$ (defined below), and the total Stand Alone charge $\sum_{N} s a\left(k_{0 i} ; k[i]\right)$.

Example 1 Consider the linear tree $0 \leftrightarrow 1 \leftrightarrow 2 \leftrightarrow \cdots \leftrightarrow n$, with connecting costs corresponding to distances, namely $k_{i j}=|i-j|$. Assume $n$ is even to fix ideas, and write $\mathcal{H}_{n}$ for the harmonic number $\mathcal{H}_{n}=\sum_{j=1}^{n} \frac{1}{j}$. We compute first the ordered costs $k_{i}^{t}$ for an arbitrary agent $i$, distinguishing two cases. For $1 \leq i \leq \frac{n}{2}$ we have

$$
k_{i}^{2 t-1}=k_{i}^{2 t}=t \text { for } 1 \leq t \leq i-1 ; k_{i}^{t^{\prime}}=t^{\prime}-i+1 \text { for } 2 i-1 \leq t^{\prime} \leq n-1
$$

Then (3) gives

$$
\begin{aligned}
\operatorname{can}_{i} & =\frac{i}{n}+\sum_{t=1}^{i-1}\left\{\frac{1}{(2 t-1) 2 t}+\frac{1}{2 t(2 t+1)}\right\} t+\sum_{t^{\prime}=2 i-1}^{n-1} \frac{1}{t^{\prime}\left(t^{\prime}+1\right)} i \\
& \Rightarrow \operatorname{can}_{i}=\sum_{j=1}^{i} \frac{1}{2 j-1}
\end{aligned}
$$

For $\frac{n}{2} \leq i \leq n$ we get

$$
\begin{aligned}
k_{i}^{2 t-1} & =k_{i}^{2 t}=t \quad \text { for } 1 \leq t \leq n-i \\
k_{i}^{t^{\prime}} & =t^{\prime}-n+i \quad \text { for } 2(n-i)+1 \leq t^{\prime} \leq n-1 \\
& \Rightarrow \operatorname{can}_{i}=\frac{i}{n}+\sum_{t=1}^{n-i}\left\{\frac{1}{(2 t-1) 2 t}+\frac{1}{2 t(2 t+1)}\right\} t+\sum_{t^{\prime}=2(n-i)+1}^{n-1} \frac{1}{t^{\prime}\left(t^{\prime}+1\right)}\left(t^{\prime}-n+i\right) \\
& \Rightarrow \operatorname{can}_{i}=\sum_{j=1}^{n-i} \frac{1}{2 j-1}+\sum_{j=2(n-i)+1}^{n} \frac{1}{j}=\mathcal{H}_{n}-\sum_{j=1}^{n-i} \frac{1}{2 j}
\end{aligned}
$$

These two equations imply for $1 \leq i \leq \frac{n}{2}$

$$
\operatorname{can}_{i}+\operatorname{can}_{n-i}=\mathcal{H}_{n}+\sum_{j=1}^{i} \frac{1}{(2 j-1) 2 j}
$$

from which we can evaluate the total charge $\sum \operatorname{can}_{i}$ when $n$ grows. For two positive sequences we use the notation $\alpha_{n} \simeq \beta_{n}$ iff $\lim _{n} \frac{\alpha_{n}}{\beta_{n}}=1$. The above computations 
imply

$$
\sum_{i=1}^{n} \operatorname{can}_{i} \simeq \frac{n}{2} \mathcal{H}_{n} \simeq \frac{1}{2} n \log n
$$

Compare the total Stand Alone charge

$$
\sum_{i=1}^{n} s a_{i}=\frac{n(n+1)}{2} \Rightarrow \frac{\sum_{i=1}^{n} \operatorname{can}_{i}}{\sum_{i=1}^{n} s a_{i}} \simeq \frac{\log n}{n}
$$

Next we note $v(N, K)=n$, so that the total canonical charge exceeds the efficient cost by the ratio $\frac{1}{2} \log n$. On the other hand it is a vanishing fraction of the cost of a uniform spanning tree, namely, the average cost $U(N, K)$ of all $(n+1)^{n-1}$ spanning trees

$$
U(N, K)=\frac{2}{n+1} \sum_{i j \in N^{0}(2)} k_{i j}
$$

In our example, using the identity $\sum_{1}^{n} i^{2}=\frac{n(n+1)(2 n+1)}{6}$, we compute

$$
U(N, K)=\frac{n(n+2)}{3} \Rightarrow \frac{\sum_{i=1}^{n} \operatorname{can}_{i}}{U(N, K)} \simeq \frac{3 \log n}{2 n}
$$

Thus the canonical charges realize most of the surplus accruing from building an optimal instead of a random uniform spanning tree.

Based on Example 1 and a couple of numerical computations, we conjecture that for any cost matrix satisfying the triangular property $k_{i j} \leq k_{i l}+k_{l j}$, the relative excess charge never grows faster than $\frac{1}{2} \log n$.

Example 2 Consider the random cost matrix $K$ where all entries $k_{i j}$ are IID with uniform distribution on $[0,1]$. Using the algorithm leading to Eq.2 it is easy to compute

$$
E\left\{\operatorname{can}_{i}\right\}=\frac{1}{n}\left(\frac{1}{2}+\frac{1}{3}+\cdots+\frac{1}{n+1}\right) \simeq \frac{\log n}{n} ; \quad \text { and } \quad E\left\{s a_{i}\right\}=\frac{1}{2}
$$

Up to a factor 2, the asymptotic behavior of $\frac{E\left\{\mathrm{can}_{i}\right\}}{E\left\{s a_{i}\right\}}$ is as in Example 1 .

Moreover Theorem 6.21 in Bollobas (2001) shows that

$$
E\{v(N, K)\} \simeq \sum_{j=1}^{\infty} \frac{1}{j^{3}} \simeq 1.202
$$


so the ratio of the charge collected by the canonical rule to the efficient one is, again, of the order $\log n$. Moreover the expected cost of a uniform spanning tree is $\frac{n}{2}$, so that $\frac{E\left\{\sum \operatorname{can}_{i}\right\}}{E\{U(N, K)\}} \simeq \frac{2 \log n}{n}$.

Notice that the choice of the uniform distribution in Example 2 is not important. Any cumulative distribution $F$ with derivative bounded away from zero $\left(F^{\prime}(x) \geq \lambda>0\right.$ for $0 \leq x \leq 1)$ gives the same asymptotic comparisons. Indeed $F^{\prime}(0)>0$ implies that the limit of $E\{v(N, K)\}$ is finite (Bollobas 2001). Setting $G=1-F$, we have $G(x) \leq 1-\lambda x$ for $0 \leq x \leq 1$. Recall that the mean of $q$ IID variables with cdf $F$ is $\int_{0}^{1} G^{q}(x) d x$, so that the algorithm leading to Eq. 2 gives

$$
E\left\{\operatorname{can}_{i}\right\}=\frac{1}{n} \sum_{q=1}^{n} \int_{0}^{1} G^{q}(x) d x \leq \frac{1}{n} \sum_{q=1}^{n} \int_{0}^{1}(1-\lambda x)^{q} d x=\frac{1}{\lambda n}\left(\mathcal{H}_{n+1}-1\right)
$$

A similar lower bound obtains if the derivative of $F$ is uniformly bounded above.

\section{Three properties of the canonical charge}

These properties play a leading role in the axiomatic discussion of the mcst and other fair division problems (see e.g., Dutta and Kar 2004; Bergantinos and Vidal-Puga 2007; Bogomolnaia and Moulin 2008). We define them directly for a decentralized pricing rule, although it is clear that they apply to general solutions, budget-balanced or not.

The first two are compelling regularity properties:

- Continuity: $f\left(N, k_{0 i}, k[i]\right)$ is continuous in $\left(k_{0 i}, k[i]\right)$.

- Cost Monotonicity: $f\left(N, k_{0 i}, k[i]\right)$ is weakly increasing in each cost $k_{i j}, k_{0 i}$.

Violation of Continuity means that a tiny measurement error may have dramatic consequences on individual charges. Violation of Cost Monotonicity opens the door to artificial inflation of one's costs.

Our third axiom compares a decentralized pricing rule across problems involving different sets of users. For any subset $S$ of $N$ we use the notation $k[i, S]$ for the $|S|$-dimensional vector $\left(k_{i j}, j \in S\right)$.

- Population Monotonicity: for any profile of costs $\left(k_{0 i}, k[i]\right)$, any $S \subset N$ and $i \in S$ we have

$$
f\left(N, k_{0 i}, k[i]\right) \leq f\left(S, k_{0 i}, k[i, S]\right) .
$$

This says that the addition of a new user is never detrimental to any of the existing users. It is a strengthening of the Stand Alone upper bound, provided we assume that in a one agent problem, the pricing rule is simply $f\left(\{i\}, k_{0 i}\right)=k_{0 i}$. Population Monotonicity generates clean incentives in the game where agents must decide whether or not to request connection to the source, based on their willingness to pay for such service (see Moulin and Shenker 2001). 
Lemma 2 The canonical pricing rule (and the Stand Alone rule) are Continuous, Cost Monotonic and Population Monotonic.

Proof All three properties are stable by fixed non negative linear combinations and the min operation. Thus it is enough to check that for all $t, 1 \leq t \leq n-1$, the mapping $\left(k_{0 i}, k[i]\right) \rightarrow k_{i}^{t}$ meets all three properties. Continuity and Cost Monotonicity are clear. For Population Monotonicity we note that the $t$ 'th element in $k[i, S]$ will be greater than or equal to the $t$ th element in $k[i]$. Hence, using (3) if removing one agent at a time and noting that $\frac{1}{n(n-1)} k_{01} \geq \frac{1}{n(n-1)} \min \left\{k_{01}, k_{1}^{n}\right\}$ we get the desired result.

\section{Main characterization}

We introduce two properties pertaining to addition and positive linear combinations of cost profiles.

- Superadditivity: for any two $\left(k_{0 i}^{1}, k^{1}[i]\right)$ and $\left(k_{0 i}^{2}, k^{2}[i]\right)$, we have

$$
f\left(N, k_{0 i}^{1}+k_{0 i}^{2}, k^{1}[i]+k^{2}[i]\right) \geq f\left(N, k_{0 i}^{1}, k^{1}[i]\right)+f\left(N, k_{0 i}^{2}, k^{2}[i]\right) .
$$

The efficient cost $v(N, K)$ is clearly superadditive in the matrix $K$ of connecting costs. Recall from Sect. 1 the interpretation of this property as a preference for flexibility. The axiom imposes the same property for the pricing rule: every participant weakly benefits from flexibility.

For our next axiom, given a permutation $\sigma$ of $\{1, \ldots, p\}$ we write $C_{\sigma}=\{x \in$ $\left.\mathbf{R}_{+}^{p} \mid x_{\sigma(1)} \leq \cdots \leq x_{\sigma(p)}\right\}$ for the cone in $\mathbf{R}_{+}^{p}$ such that the relative ordering of the coordinates is constant and given by $\sigma$. We say that the real valued function $g$ with domain $\mathbf{R}_{+}^{p}$ is piece-wise linear if for any $\sigma$ its restriction to $C_{\sigma}$ is positively linear (respects positive linear combinations). Key observation: the efficient $\operatorname{cost} v(N, K)$ is piece-wise linear in $\mathbf{R}_{+}^{\frac{n(n+1)}{2}}$.

- Piece-wise Linearity: the pricing rule $\left(k_{0 i}, k[i]\right) \rightarrow f\left(k_{0 i}, k[i]\right)$ is piece-wise linear in $\mathbf{R}_{+}^{n}$.

Like Superadditivity above (or additivity in the axiomatic cost sharing literature), this axiom wants the solution to share some structural property of the efficient cost. Its main justification is informational parsimony: a piece-wise linear pricing rule is entirely determined by its value over $n$ coordinate vectors in one of the cones $C_{\sigma}$.

Theorem 1 The canonical pricing rule (and the Stand Alone rule), are superadditive and piece-wise linear.

Conversely, if a decentralized pricing rule $f$ is superadditive or piece-wise linear, we have

$\left\{f\left(k_{0 i}, k[i]\right) \leq s a\left(k_{0 i}, k[i]\right)\right.$ for all $\left.K\right\} \Rightarrow\left\{\operatorname{can}\left(k_{0 i}, k[i]\right) \leq f\left(k_{0 i}, k[i]\right)\right.$ for all $\left.K\right\}$.

Proof Step 1. can is superadditive and piece-wise linear 
In the sum (2) defining $\operatorname{can}\left(k_{0 i} ; k[i]\right)$, each term $\min _{j \in \mathcal{P}(i, \pi)}\left\{k_{i j}\right\}$ is superadditive in $\left(k_{0 i}, k[i]\right)$. If the relative ordering of the $n$ numbers $\left(k_{0 i}, k_{i j}, j \in N \backslash\{i\}\right)$ remains fixed, each term $\min _{j \in \mathcal{P}(i, \pi)}\left\{k_{i j}\right\}$ is positively linear in $\left(k_{0 i}, k[i]\right)$.

Step 2. Assume $f$ is superadditive and $f \leq s a$, prove can $\leq f$

Notation: we write $\gamma^{t}$ for the $n$-dimensional vector $\gamma^{t}=(1 ; \overbrace{0, \ldots, 0, \overbrace{1, \ldots, 1}^{t-1}}^{n-t})$. Fix $x \geq 0$ and consider the cost matrix $K$ with $k_{i j}=k_{0 i}=x$ for all $i, j$. Feasibility implies $n x \leq n f\left(x \gamma^{1}\right)$; moreover by assumption $f\left(x \gamma^{1}\right) \leq s a\left(x \gamma^{1}\right)=x$. Thus $f\left(x \gamma^{1}\right)=x$. Next fix any $t, 2 \leq t \leq n-1$, and consider the cost matrix

$$
k_{0 i}=x \text { for all } i ; k_{i j}=0 \text { if } 1 \leq i, j \leq t ; k_{i j}=x \text { otherwise. }
$$

Observe that the canonical price is $f\left(x \gamma^{1}\right)=x$ for agents $t+1, \ldots, n$ and $f\left(x \gamma^{t}\right)$ for agents $1, \ldots, t$. Feasibility implies

$$
t f\left(x \gamma^{t}\right)+(n-t) x \geq(n-t+1) x \Rightarrow f\left(x \gamma^{t}\right) \geq \frac{x}{t}
$$

Next we pick a $n$-dimensional vector of costs $\left(a ; b_{2}, b_{3}, . ., b_{n}\right)$, where $a$ is the cost to the source, and

$$
b_{2} \leq \cdots \leq b_{p} \leq a \leq b_{(p+1)} \leq \cdots \leq b_{n}
$$

We decompose the vector $\left(a ; b_{2}, b_{3}, . ., b_{n}\right)$ as follows

$$
\begin{gathered}
\left(a ; b_{2}, b_{3}, . ., b_{n}\right)=b_{2} \gamma^{1}+\left\{\sum_{t=2}^{p-1}\left(b_{t+1}-b_{t}\right) \gamma^{t}\right\}+\left(a-b_{p}\right) \gamma^{p} \\
+(0 ; \overbrace{0, \ldots, 0}^{p-1}, b_{(p+1)}-a \ldots, b_{n}-a)
\end{gathered}
$$

By the argument above

$$
\begin{aligned}
f\left(b_{2} \gamma^{1}\right) & =b_{2} ; f\left(\left(b_{t+1}-b_{t}\right) \gamma^{t}\right) \\
& \geq \frac{1}{t}\left(b_{t+1}-b_{t}\right) \text { for } 2 \leq t \leq p-1 ; f\left(\left(a-b_{p}\right) \gamma^{p}\right) \geq \frac{1}{p}\left(a-b_{p}\right)
\end{aligned}
$$

Now superadditivity and $f \geq 0$ imply

$$
f\left(a ; b_{2}, b_{3}, . ., b_{n}\right) \geq b_{2}+\frac{\left(b_{3}-b_{2}\right)}{2}+\frac{\left(b_{4}-b_{3}\right)}{3}+\cdots+\frac{\left(a-b_{p}\right)}{p}
$$

the desired conclusion, upon checking that the right-hand-side is precisely $\operatorname{can}\left(a ; b_{2}, b_{3}, . ., b_{n}\right)$.

Step 3. Assume $f$ is piece-wise linear and $f \leq s a$, prove can $\leq f$ 
Both sides of the desired inequality are piece-wise linear in $\left(k_{0 i}, k[i]\right)$ and symmetric in $k[i]$. Thus it is enough to prove can $\left(a ; b_{2}, b_{3}, . ., b_{n}\right) \leq f\left(a ; b_{2}, b_{3}, . ., b_{n}\right)$ when $\left(a ; b_{2}, b_{3}, . ., b_{n}\right)$ is in one of the cones $C^{p}$ defined by (5) for $2 \leq p \leq n$, or in $C^{1}$ defined by $a \leq b_{2} \leq \cdots \leq b_{n}$. Fix $p, 2 \leq p \leq n$. By assumption, in $C^{p}$ the function $f$ takes the form

$$
f(a ; b)=\lambda_{1} a+\sum_{s=2}^{n} \lambda_{s} b_{s}
$$

for some fixed numbers $\lambda_{s}$ (depending on $p$ ).

We use the notation $\gamma^{t}$ in step 1 , as well as $\delta^{t}=(0 ; \overbrace{0, \ldots, 0}^{t-1}, \overbrace{1, \ldots, 1}^{n-t})$. Note that $\gamma^{t} \in C^{p}$ iff $t \leq p$, while $\delta^{t} \in C^{p}$ iff $t \geq p$.

From the stand alone upper bound at $\delta^{t}$ for $t \geq p$, we get $\sum_{s=t+1}^{n} \lambda_{s} \leq 0$. Our assumption that $f$ is non negative then implies $\lambda_{s}=0$ for $s \geq p+1$. $^{6}$

Next we consider the cost matrix $k_{i j}=k_{0 i}=1$ for all $i, j$ : feasibility and $f \leq s a$ imply $f\left(\gamma^{1}\right)=1 \Leftrightarrow \sum_{s=1}^{p} \lambda_{s}=1$.

Finally, we fix $t \leq p$ and consider the cost matrix $K^{t}$

$$
\begin{gathered}
k_{0 i}=1 \text { for all } i ; k_{i j}=0 \text { if } i, j \leq t, k_{i j}=1 \text { otherwise } \\
\Rightarrow\left(k_{0 i}, k[i]\right)=\gamma^{t} \text { if } i \leq t ;\left(k_{0 i}, k[i]\right)=\gamma^{1} \text { if } i \geq t+1
\end{gathered}
$$

Here feasibility gives

$$
\begin{aligned}
v\left(N, K^{t}\right) & =n-t+1 \leq t f\left(\gamma^{t}\right)+(n-t) f\left(\gamma^{1}\right)=t\left(\lambda_{1}+\sum_{s=t+1}^{p} \lambda_{s}\right)+(n-t) \\
& \Leftrightarrow f\left(\gamma^{t}\right) \geq \frac{1}{t}=\operatorname{can}\left(\gamma^{t}\right) \text { for } 1 \leq t \leq p
\end{aligned}
$$

In $C^{p}$, both $f(a ; b)$ and $\operatorname{can}(a ; b)$ are linear combinations of $\left(a ; b_{2}, \ldots, b_{p}\right)$ only (recall $\lambda_{s}=0$ for $s \geq p+1$ ), and each vector in the cone $\left\{b_{2} \leq \cdots \leq b_{p} \leq a\right\}$ is a positive linear combination of the vectors $\gamma^{t}, 1 \leq t \leq p$. Therefore the above inequalities conclude the proof.

Example 3 Here is a three agent example of a superadditive and piece-wise linear decentralized pricing rule not bounded below by the canonical rule. Let

$$
f\left(a ; b_{1}, b_{2}\right)=\frac{1}{3} a+\min \left\{b_{1}, b_{2}\right\}
$$

6 Note that even absent the assumption $f \geq 0$, these equalities follow the feasibility property (1). 
Check that inequality (1) holds:

$$
f\left(a ; b_{1}, b_{2}\right)+f\left(a^{\prime} ; b_{2}, b_{3}\right)+f\left(a^{\prime \prime} ; b_{1}, b_{3}\right)=\bar{a}+2 b^{1}+b^{2}
$$

where $\bar{a}$ is the mean of $a, a^{\prime}, a^{\prime \prime}$, and $b^{1}, b^{2}$ are the two smallest values among $b_{1}, b_{2}, b_{3}$. This is clearly bounded below by $v(N, K)$. It is just as easy to check that $f$ is not bounded above by the Stand Alone rule, and that it is continuous and cost monotonic. Finally, we have

$$
f(4 ; 1,5)=\frac{7}{3}<\operatorname{can}(4 ; 1,5)=\frac{1}{3} 4+\frac{1}{2}+\frac{1}{6} 4=\frac{5}{2} .
$$

\section{A direct characterization}

We report an additional characterization of the canonical pricing rule, based on the following two axioms on pricing rules.

- Independence of Irrelevant Links: for any two agents $i, j \in N$ and profile of costs $\left(k_{0 i}, k[i]\right)$

$$
\left\{k_{i j} \geq k_{0 i}\right\} \Rightarrow f\left(N, k_{0 i}, k[i]\right)=f\left(N \backslash\{j\}, k_{0 i}, k[i, N \backslash\{j\}]\right) .
$$

If $k_{i j} \geq k_{0 i}$, the $i j$ link is not relevant for agent $i$ when linking to any network. Independence of Irrelevant Links then stipulates that the irrelevant cost $k_{i j}$ has no impact on the price charged to agent $i$.

Next we have a decentralized version of an axiom analyzed in Bergantinos and Vidal-Puga (2007):

- Equal Share of Extra Costs: for any profile of costs $\left(k_{0 i}, k[i]\right)$, agent $i$ and number $\delta>0$

$$
\left\{k_{i j} \leq k_{0 i} \text { for all } j\right\} \Rightarrow f\left(k_{0 i}+\delta, k[i]\right)=f\left(k_{0 i}, k[i]\right)+\frac{\delta}{n} \text { for all } i
$$

This axiom applies only to profiles where all nodes $j$ are relevant to $i$ 's charge, because it is cheaper to connect to any one of them than to the source. Then it requires to charge to agent $i$ a fair share of any additional cost of connecting to the source.

Theorem 2 The Canonical Pricing Rule is uniquely characterized by Independence of Irrelevant Links, Equal Share of Extra Costs, and the stand alone upper bound: $f\left(k_{0 i}, k[i]\right) \leq k_{0 i}$.

Proof (Sketch) It is easy to check that the axioms are satisfied by can. We show that they lead to a unique solution. By Independence of Irrelevant Links all agents with higher link costs than $i$ 's source cost can be disregarded. Next, among the remaining agents, rank their link costs in increasing order and consider the highest cost $k^{*}$ below $k_{0 i}$. By Equal Share of Extra Costs the difference $k^{*}-k_{0 i}$ is shared equally between the remaining agents. Using Independence of Irrelevant Links the agent(s) with link 
cost $k^{*}$ can then be removed and Equal Share of Extra Costs can be used to share the difference between $k^{*}$ and the second highest cost and so forth until only agent $i$ is left to be connected to the source. By feasibility and stand alone upper bound agent $i$ must then pay his source cost. Thus, the price is uniquely determined by the axioms.

We note that in the special case of two-agent pricing problems the canonical pricing rule is uniquely characterized by feasibility (1), Stand Alone upper bound and Equal Share of Extra Costs. We omit the straightforward argument.

\section{References}

Aarts, H., Driessen, T.: The irreducible core of a minimum cost spanning tree game. ZOR Methods Models Oper Res 38, 163-174 (1993)

Bergantinos, G., Vidal-Puga, J.J.: A fair rule in minimum cost spanning tree problems. J Econ Theory 137, 326-352 (2007)

Bird, C.G.: On cost allocation for a spanning tree: a game theoretic approach. Networks 6, 335-350 (1976)

Bogomolnaia, A., Moulin, H.: A new solution to the random assignment problem. J Econ Theory 100, 295$328(2001)$

Bogomolnaia, A., Moulin, H.: Sharing the cost of a minimal cost spanning tree: beyond the Folk solution, mimeo, Rice University (2008)

Brânzei, R., Moretti, S., Norde, H., Tijs, S.: The $P$-value for cost sharing in minimum cost spanning tree situations. Theory Dec 56, 47-61 (2004)

Brams, S.J., Taylor, A.D.: Fair Division: from Cake-cutting to Dispute Resolution. Cambridge: Cambridge University Press (1996)

Bollobas, B.: Random Graphs. 2nd edn. Cambridge: Cambridge University Press (2001)

Claus, A., Kleitman, D.J.: Cost allocation for a spanning tree. Networks 3, 289-304 (1973)

Demko, S., Hill, T.P.: Equitable distribution of indivisible objects. Math Soc Sci 16, 145-158 (1988)

Dubins, L.F.: Group decision devices, American Math Monthly, May, 350-356 (1977)

Dutta, B., Kar, A.: Cost monotonicity, consistency, and minimum cost spanning tree games. Games Econ Behav 48, 223-248 (2004)

Feltkamp, T., Tijs, S., Muto, S.: On the irreducible core and the equal remaining obligations rule of minimum cost spanning extension problems, mimeo University of Tilburg, CentER DP 94106 (1994)

Kruskal, J.B.: On the shortest spanning subtree of a graph and the traveling salesman problem. Proc Am Math Soc 7, 48-50 (1956)

Moulin, H.: Characterizations of the pivotal mechanism. J Pub Econ 31, 53-78 (1986)

Moulin, H.: Uniform externalities: two axioms for fair allocation. J Pub Econ 43, 305-326 (1990)

Moulin, H.: Welfare bounds in the cooperative production problem. Games Econ Behav 4, 373-401 (1992)

Moulin, H., Shenker, S.: Strategyproof sharing of submodular costs: budget balance versus efficiency. Econ Theory 18, 511-533 (2001)

Norde, H., Moretti, S., Tijs, S.: Minimum cost spanning tree games and population monotonic allocation schemes. Euro J Oper Res 154, 84-97 (2001)

Prim, R.C.: Shortest connection networks and some generalizations. Bell Sys Tech J 36, 1389-1401 (1957)

Sharkey, W.W.: The Theory of Natural Monopoly. Cambridge: Cambridge University Press (1982)

Sharkey, W.W.: Network models in economics. In: Ball et al. (eds.) Handbooks in Operations Research and Management Science, pp. 713-765. New York: Elsevier (1995)

Steinhaus, H.: The problem of fair division. Econometrica 16, 101-104 (1948)

Thomson, W.: Maximin Strategies and elicitation of preferences. In: Laffont, J.J. Aggregation and Revelation of Preferences, Studies in Public economics, Amsterdam: North-Holland (1979) 